\title{
Factorizations for a Class of Multivariate Polynomial Matrices
}

\author{
Dong Lu ${ }^{\mathrm{a}, \mathrm{b}}$, Dingkang Wang, ${ }^{\mathrm{a}, \mathrm{b}}$, Fanghui Xiao ${ }^{\mathrm{a}, \mathrm{b}}$ \\ ${ }^{a}$ KLMM, Academy of Mathematics and Systems Science, Chinese Academy of Sciences, Beijing 100190, China \\ ${ }^{b}$ School of Mathematical Sciences, University of Chinese Academy of Sciences, Beijing 100049, China
}

\begin{abstract}
Following the works by Lin et al. (Circuits Syst. Signal Process. 20(6): 601-618, 2001) and Liu et al. (Circuits Syst. Signal Process. 30(3): 553-566, 2011), we investigate how to factorize a class of multivariate polynomial matrices. The main theorem in this paper shows that an $l \times m$ polynomial matrix admits a factorization with respect to a polynomial if the polynomial and all the $(l-1) \times(l-1)$ reduced minors of the matrix generate the unit ideal. This result is a further generalization of previous works, and based on this, we give an algorithm which can be used to factorize more polonomial matrices. In addition, an illustrate example is given to show that our main theorem is non-trivial and valuable.
\end{abstract}

Keywords: Multivariate polynomial matrices, Matrix factorizations, Reduced minors, Reduced Gröbner basis

\section{Introduction}

The study of factorizations for multivariate polynomial matrices began with the development of multidimensional system theory in the late 1970s (Youla and Gnavi, 1979), and the problem of matrix factorizations was considered to be one of the basic problems of this subject. Since then, great progress has been made on multivariate polynomial matrix factorizations.

Bose (1982) introduced some basic concepts of multivariate polynomial matrices and the problem of matrix factorizations. After that, Bose et al. (2003) presented factorization algorithms of bivariate polynomial matrices, and introduced the latest research trends of matrix factorizations with three or more variables. The factorization problem for bivariate polynomial matrices has been completely solved in (Guiver and Bose, 1982; Liu and Wang, 2013; Morf et al., 1977), but for the cases of more than two variables is still open.

Charoenlarpnopparut and Bose (1999) used Gröbner bases of modules to compute zero prime matrix factorizations of multivariate polynomial matrices. For some polynomial matrices with special properties, Lin $(1999 a, 2001)$ proposed some methods to compute zero prime matrix factorizations of matrices. Meanwhile, Lin and Bose (2001) presented the Lin-Bose's conjecture: a matrix admits a zero prime matrix factorization if its all maximal reduced minors generate the unit ideal. This conjecture was proved in (Liu et al., 2014; Pommaret, 2001; Wang and Feng, 2004), so the problem of zero prime matrix factorizations have been completely solved. Subsequently, Wang and Kwong (2005) put forward an algorithm based on module theory to solve the problem

Email addresses: donglu@amss.ac.cn (Dong Lu), dwang@mmrc.iss.ac.cn (Dingkang Wang), xiaofanghui@amss.ac.cn (Fanghui Xiao) 
of minor prime matrix factorizations. Guan et al. $(2018,2019)$ studied the problem of factor prime matrix factorizations under the condition that matrices are not of full rank, and they generalized the main results in (Wang and Kwong, 2005). So far, some achievements in (Liu and Wang, 2010, 2015; Wang, 2007) have been made on the problem of factor prime matrix factorizations. Although the problems of zero prime matrix factorizations and minor prime matrix factorizations have been completely solved, the problem of factor prime matrix factorizations remains to be studied.

Let $k[\mathbf{z}]$ and $k\left[\mathbf{z}_{2}\right]$ be the ring of polynomials in variables $z_{1}, z_{2}, \ldots, z_{n}$ and $z_{2}, \ldots, z_{n}$ with coefficients in an algebraically closed field $k$, respectively. Let $\mathbf{F}$ be an $l \times m$ polynomial matrix with entries in $k[\mathbf{z}]$ and $l \leq m, d_{l}(\mathbf{F})$ be the greatest common divisor of all the $l \times l$ minors of $\mathbf{F}$, and $d=z_{1}-f\left(\mathbf{z}_{2}\right)$ be a divisor of $d_{l}(\mathbf{F})$, where $f\left(\mathbf{z}_{2}\right) \in k\left[\mathbf{z}_{2}\right]$. Lin et al. (2001) proved that $\mathbf{F}$ admits a matrix factorization with respect to $d$ if for each $\left(\mathbf{z}_{2}\right) \in k^{1 \times(n-1)}$ the rank of $\mathbf{F}\left(f\left(\mathbf{z}_{2}\right), \mathbf{z}_{2}\right)$ is $(l-1)$. Moreover, they proposed a constructive algorithm to factorize this class of multivariate polynomial matrices. Liu et al. (2011) focused on the relationship between $d$ and all the $(l-1) \times(l-1)$ minors of $\mathbf{F}$, and showed that $\mathbf{F}$ admits a matrix factorization with respect to $d$ if $d$ and all the $(l-1) \times(l-1)$ minors of $\mathbf{F}$ generate $k[\mathbf{z}]$. They proved that their main theorem is a generalization of the result in (Lin et al., 2001). However, we find that there are still many of multivariate polynomial matrices that can be factorized with respect to $d$ without satisfying the main theorem in (Liu et al., 2011). This implies that it would be significant to generalize the theorems and algorithms in (Lin et al., 2001; Liu et al., 2011).

In this paper, we still study the condition under which $\mathbf{F}$ admits a matrix factorization with respect to $d$. We focus on the relationship between $d$ and all the $(l-1) \times(l-1)$ reduced minors of $\mathbf{F}$, and prove that $\mathbf{F}$ admits a matrix factorization with respect to $d$ if $d$ and all the $(l-1) \times(l-1)$ reduced minors of $\mathbf{F}$ generate the unit ideal. Compared with the main theorems in (Lin et al., 2001; Liu et al., 2011), our main theorem has a wider range of applications. Combining our main theorem and the constructive algorithm in (Lin et al., 2001), we obtain the matrix factorization algorithm.

This paper is organized as follows. In Section 2, we outline some knowledge about multivariate polynomial matrix factorizations and propose a problem that we shall consider. Main theorem and some generalizations are presented in Section 3 to help us summarize which types of polynomial matrices can be factorized. The matrix factorization algorithm is given in Section 4 , and an example is given to illustrate the calculation process of the algorithm. Further remarks are provided in Section 5 .

\section{Preliminaries and Problem}

In the following, we denote by $k$ an algebraically closed field, $\mathbf{z}$ the $n$ variables $z_{1}, z_{2}, \ldots, z_{n}$, $\mathbf{z}_{2}$ the $(n-1)$ variables $z_{2}, \ldots, z_{n}$, where $n \geq 3$. Let $k[\mathbf{z}]$ and $k\left[\mathbf{z}_{2}\right]$ be the ring of polynomials in variables $\mathbf{z}$ and $\mathbf{z}_{2}$ with coefficients in $k$, respectively, $k(\mathbf{z})$ be the fraction field of $k[\mathbf{z}]$, and $k[\mathbf{z}]^{l \times m}$ be the set of $l \times m$ matrices with entries in $k[\mathbf{z}]$. Without loss of generality, we assume that $l \leq m$, and for convenience we use uppercase bold letters to denote polynomial matrices.

Throughout this paper, the argument $(\mathbf{z})$ is omitted whenever its omission does not cause confusion. For any given $\mathbf{F} \in k[\mathbf{z}]^{l \times m}$ and $f\left(\mathbf{z}_{2}\right) \in k\left[\mathbf{z}_{2}\right], \mathbf{F}^{\mathrm{T}}$ represents the transposed matrix of $\mathbf{F}$, and $\mathbf{F}\left(f\left(\mathbf{z}_{2}\right), \mathbf{z}_{2}\right)$ denotes an $l \times m$ polynomial matrix in $k\left[\mathbf{z}_{2}\right]^{l \times m}$, which is formed by transforming $z_{1}$ in $\mathbf{F}$ into $f\left(\mathbf{z}_{2}\right)$. If $l=m$, we denote by $\operatorname{det}(\mathbf{F})$ the determinant of $\mathbf{F}$, and if $\mathbf{F}$ is of full rank, we use $\mathbf{F}^{-1} \in k(\mathbf{z})^{l \times l}$ to stand for the inverse matrix of $\mathbf{F}$. Assume that $f_{1}, \ldots, f_{s} \in k[\mathbf{z}]$, we use $\left\langle f_{1}, \ldots, f_{s}\right\rangle$ to denote the ideal generated by $f_{1}, \ldots, f_{s}$ in $k[\mathbf{z}]$. Let $f, g \in k[\mathbf{z}]$, then $f \mid g$ means 
that $f$ is a divisor of $g$. In addition, "w.r.t." and "GCD" stand for "with respect to" and "greatest common divisor", respectively.

\subsection{Previous Works}

We first introduce two basic concepts in matrix theory.

Definition 2.1. Let $\mathbf{F} \in k[\mathbf{z}]^{l \times m}$, and given $2 r$ positive integers arbitrarily such that $1 \leq i_{1}<$ $\cdots<i_{r} \leq l$ and $1 \leq j_{1}<\cdots<j_{r} \leq m$. Let $\mathbf{F}\left(\begin{array}{c}i_{1} \cdots i_{r} \\ j_{1} \cdots j_{r}\end{array}\right)$ denotes an $r \times r$ matrix consisting of the $i_{1}, \ldots, i_{r}$ rows and $j_{1}, \ldots, j_{r}$ columns of $\mathbf{F}$, then $\operatorname{det}\left(\mathbf{F}\left(\begin{array}{c}i_{1} \cdots i_{r} \\ j_{1} \cdots j_{r}\end{array}\right)\right)$ is called an $r \times r$ minor of $\mathbf{F}$.

Definition 2.2. Let $\mathbf{F} \in k[\mathbf{z}]^{l \times m}$, the rank of $\mathbf{F}$ is $r(1 \leq r \leq l)$ if there exists a nonzero $r \times r$ minor of $\mathbf{F}$, and all the $i \times i(i>r)$ minors of $\mathbf{F}$ vanish identically. For convenience, we denote the rank of $\mathbf{F}$ by $\operatorname{rank}(\mathbf{F})$.

The following lemma is a generalization of Binet-Cauchy formula in (Strang, 2010).

Lemma 2.3. Let $\mathbf{F}=\mathbf{G}_{1} \mathbf{F}_{1} \in k[\mathbf{z}]^{l \times m}$, where $\mathbf{G}_{1} \in k[\mathbf{z}]^{l \times l}$ and $\mathbf{F}_{1} \in k[\mathbf{z}]^{l \times m}$. Then an $r \times r$ $(r \leq l)$ minor of $\mathbf{F}$ is

$$
\operatorname{det}\left(\mathbf{F}\left(\begin{array}{l}
i_{1} \cdots i_{r} \\
j_{1} \cdots j_{r}
\end{array}\right)\right)=\sum_{1 \leq s_{1}<\cdots<s_{r} \leq l} \operatorname{det}\left(\mathbf{G}_{1}\left(\begin{array}{c}
i_{1} \cdots i_{r} \\
s_{1} \cdots s_{r}
\end{array}\right)\right) \cdot \operatorname{det}\left(\mathbf{F}_{1}\left(\begin{array}{c}
s_{1} \cdots s_{r} \\
j_{1} \cdots j_{r}
\end{array}\right)\right)
$$

In particular, when $r=l$, we have

$$
\operatorname{det}\left(\mathbf{F}\left(\begin{array}{ccl}
1 & \cdots l \\
j_{1} \cdots j_{l}
\end{array}\right)\right)=\operatorname{det}\left(\mathbf{G}_{1}\right) \cdot \operatorname{det}\left(\mathbf{F}_{1}\left(\begin{array}{ccl}
1 & \cdots l \\
j_{1} \cdots j_{l}
\end{array}\right)\right) .
$$

To make the description simpler, we use the notations and concepts in the paper (Lin, 1988).

Definition 2.4. For any given $\mathbf{F} \in k[\mathbf{z}]^{l \times m}$,

1. let $d_{l}(\mathbf{F})$ and $d_{l-1}(\mathbf{F})$ be the $G C D$ of all the $l \times l$ minors and all the $(l-1) \times(l-1)$ minors of $\mathbf{F}$, respectively;

2. let $a_{1}, \ldots, a_{\eta} \in k[\mathbf{z}]$ be all the $l \times l$ minors of $\mathbf{F}$, where $\eta=\left(\begin{array}{c}m \\ l\end{array}\right)$, and extracting $d_{l}(\mathbf{F})$ from $a_{1}, \ldots, a_{\eta}$ yields

$$
a_{i}=d_{l}(\mathbf{F}) b_{i}, \quad i=1, \ldots, \eta,
$$

then $b_{1}, \ldots, b_{\eta}$ are called the $l \times l$ reduced minors of $\mathbf{F}$;

3. let $c_{1}, \ldots, c_{\gamma} \in k[\mathbf{z}]$ be all the $(l-1) \times(l-1)$ minors of $\mathbf{F}$, where $\gamma=\left(\begin{array}{c}l \\ l-1\end{array}\right) \cdot\left(\begin{array}{c}m \\ l-1\end{array}\right)$, and extracting $d_{l-1}(\mathbf{F})$ from $c_{1}, \ldots, c_{\gamma}$ yields

$$
c_{i}=d_{l-1}(\mathbf{F}) h_{i}, \quad i=1, \ldots, \gamma,
$$

then $h_{1}, \ldots, h_{\gamma}$ are called the $(l-1) \times(l-1)$ reduced minors of $\mathbf{F}$.

Now, we introduce two important lemmas in matrix theory.

Lemma 2.5 (Lin $(1993,1999 b))$. Let $\mathbf{F}_{1}=\left[\mathbf{F}_{11}, \mathbf{F}_{12}\right] \in k[\mathbf{z}]^{l \times(m+l)}$ be of full row rank and $\mathbf{F}_{2}=$ $\left[\mathbf{F}_{21}^{\mathrm{T}},-\mathbf{F}_{22}^{\mathrm{T}}\right]^{\mathrm{T}} \in k[\mathbf{z}]^{(m+l) \times m}$ be of full column rank, where $\mathbf{F}_{11}, \mathbf{F}_{22} \in k[\mathbf{z}]^{l \times m}, \mathbf{F}_{12} \in k[\mathbf{z}]^{l \times l}$ and $\mathbf{F}_{21} \in k[\mathbf{z}]^{m \times m}$. If $\mathbf{F}_{1} \mathbf{F}_{2}=\mathbf{0}_{l \times m}$, then $\operatorname{det}\left(\mathbf{F}_{12}\right) \neq 0$ if and only if $\operatorname{det}\left(\mathbf{F}_{21}\right) \neq 0$. 
Lemma 2.6 (Lin (1988)). Assume that $\mathbf{F}_{12}^{-1} \mathbf{F}_{11}=\mathbf{F}_{22} \mathbf{F}_{21}^{-1}$, where $\mathbf{F}_{11}, \mathbf{F}_{22} \in k[\mathbf{z}]^{l \times m}, \mathbf{F}_{12}^{-1} \in$ $k(\mathbf{z})^{l \times l}$ and $\mathbf{F}_{21}^{-1} \in k(\mathbf{z})^{m \times m}$. Let $\bar{p}_{1}, \ldots, \bar{p}_{\xi_{1}}$ be all the $l \times l$ reduced minors of $\left[\mathbf{F}_{11}, \mathbf{F}_{12}\right]$, and $p_{1}, \ldots, p_{\xi_{2}}$ be all the $m \times m$ reduced minors of $\left[\mathbf{F}_{21}^{\mathrm{T}},-\mathbf{F}_{22}^{\mathrm{T}}\right]^{\mathrm{T}}$, where $\xi_{1}=\left(\begin{array}{c}m+l \\ l\end{array}\right)=\xi_{2}=\left(\begin{array}{c}m+l \\ m\end{array}\right)$. Then, $\bar{p}_{i}= \pm p_{i}$ for $i=1, \ldots, \xi_{1}$, and the sign depends on the index $i$.

The general matrix factorization problem is now formulated as follows.

Definition 2.7. Let $\mathbf{F} \in k[\mathbf{z}]^{l \times m}$ and $d \in k[\mathbf{z}]$ be a divisor of $d_{l}(\mathbf{F})$. We say that $\mathbf{F}$ admits a matrix factorization w.r.t. $d$ if $\mathbf{F}$ can be factorized as

$$
\mathbf{F}=\mathbf{G}_{1} \mathbf{F}_{1}
$$

such that $\mathbf{G}_{1} \in k[\mathbf{z}]^{l \times l}, \mathbf{F}_{1} \in k[\mathbf{z}]^{l \times m}$, and $\operatorname{det}\left(\mathbf{G}_{1}\right)=d$.

Next we recall the concept of zero left prime matrix from multidimensional systems theory.

Definition 2.8. Let $\mathbf{F} \in k[\mathbf{z}]^{l \times m}$ be of full row rank. If all the $l \times l$ minors of $\mathbf{F}$ generate $k[\mathbf{z}]$, then $\mathbf{F}$ is said to be a zero left prime (ZLP) matrix.

In Definition 2.7 if $\mathbf{F}_{1}$ is a ZLP matrix, then we say that $\mathbf{F}$ admits a ZLP matrix factorization. Let $I$ be an ideal generated by all the $l \times l$ minors of $\mathbf{F}$, then we can compute the reduced Gröbner basis $\mathcal{G}$ of $I$ w.r.t. a term order to check $I=k[\mathbf{z}]$. That is, if $\mathcal{G}=\{1\}$, then $I=k[\mathbf{z}]$. The definition of reduced Gröbner basis and how to compute a reduced Gröbner basis of an ideal can be found in (Buchberger, 1965; Cox et al., 2007).

Serre (1955) raised the question whether any finitely generated projective module over a polynomial ring is free. This question was solved positively and independently by Quillen (1976) and Suslin (1976), and the result is called Quillen-Suslin theorem. For Quillen-Suslin theorem, there are two descriptions as follows.

Lemma 2.9. If $\mathbf{w} \in k[\mathbf{z}]^{1 \times l}$ is a ZLP vector, then the set $\mathbf{M} \subset k[\mathbf{z}]^{l \times 1}$ constructed by all solutions $\mathbf{q} \in k[\mathbf{z}]^{l \times 1}$ of $\mathbf{w q}=0$ is free.

Lemma 2.10. If $\mathbf{w} \in k[\mathbf{z}]^{1 \times l}$ is a ZLP vector, then an unimodular matrix $\mathbf{U} \in k[\mathbf{z}]^{l \times l}$ can be constructed such that $\mathbf{w}$ is its first row.

In Lemma 2.9, $\mathbf{M}$ is called the syzygy module of $\mathbf{w}$. Fabiańska and Quadrat (2006) gave an algorithm to compute free bases of free modules over polynomial rings, and the algorithm was implemented in QuillenSuslin package (Fabiańska and Quadrat, 2007). In Lemma 2.10, U is an unimodular matrix if and only if $\operatorname{det}(\mathbf{U})$ is a nonzero constant in $k$. There are many methods to construct $\mathbf{U}$ such that $\mathbf{w}$ is its first row, we refer to (Logar and Sturmfels, 1992; Lu et al., 2017; Park, 1995; Youla and Pickel, 1984) for more details.

\subsection{Problem}

In order to raise the problem we are going to consider, let us first introduce the works in (Lin et al., 2001) and (Liu et al., 2011).

Lemma 2.11 (Lin et al. (2001)). Let $\mathbf{F} \in k[\mathbf{z}]^{l \times m}$, and $d=z_{1}-f\left(\mathbf{z}_{2}\right)$ be a common divisor of $a_{1}, \ldots, a_{\eta}$, i.e., $a_{i}=d e_{i}$ with $e_{i} \in k[\mathbf{z}](i=1, \ldots, \eta)$. If $\left\langle d, e_{1}, \ldots, e_{\eta}\right\rangle=k[\mathbf{z}]$, then $\operatorname{rank}\left(\mathbf{F}\left(f\left(\mathbf{z}_{2}\right), \mathbf{z}_{2}\right)\right)=l-1$ for every $\left(\mathbf{z}_{2}\right) \in k^{1 \times(n-1)}$ and $\mathbf{F}$ admits a matrix factorization w.r.t. d. 
Liu et al. (2011) proved that $\operatorname{rank}\left(\mathbf{F}\left(f\left(\mathbf{z}_{2}\right), \mathbf{z}_{2}\right)\right)=l-1$ for every $\left(\mathbf{z}_{2}\right) \in k^{1 \times(n-1)}$ if and only if $\left\langle d, c_{1}, \ldots, c_{\gamma}\right\rangle=k[\mathbf{z}]$. Therefore, they generalized Lemma 2.11 and obtained the following result.

Lemma 2.12 (Liu et al. (2011)). Let $\mathbf{F} \in k[\mathbf{z}]^{l \times m}$, and $d=z_{1}-f\left(\mathbf{z}_{2}\right)$ be a divisor of $d_{l}(\mathbf{F})$. If $\left\langle d, c_{1}, \ldots, c_{\gamma}\right\rangle=k[\mathbf{z}]$, then $\operatorname{rank}\left(\mathbf{F}\left(f\left(\mathbf{z}_{2}\right), \mathbf{z}_{2}\right)\right)=l-1$ for every $\left(\mathbf{z}_{2}\right) \in k^{1 \times(n-1)}$ and $\mathbf{F}$ admits a matrix factorization w.r.t. d.

In the following, let $d=z_{1}-f\left(\mathbf{z}_{2}\right)$ with $f\left(\mathbf{z}_{2}\right) \in k\left[\mathbf{z}_{2}\right]$. According to Lemma 2.11 and Lemma 2.12, we construct two sets of multivariate polynomial matrices:

$$
\begin{cases}\mathcal{S}_{1} & :=\left\{\mathbf{F} \in k[\mathbf{z}]^{l \times m}: d \mid d_{l}(\mathbf{F}) \text { and }\left\langle d, e_{1}, \ldots, e_{\eta}\right\rangle=k[\mathbf{z}]\right\} \\ \mathcal{S}_{2} & :=\left\{\mathbf{F} \in k[\mathbf{z}]^{l \times m}: d \mid d_{l}(\mathbf{F}) \text { and }\left\langle d, c_{1}, \ldots, c_{\gamma}\right\rangle=k[\mathbf{z}]\right\}\end{cases}
$$

Then, we have $\mathcal{S}_{1} \subset \mathcal{S}_{2}$ and $\mathbf{F} \in \mathcal{S}_{2}$ admits a matrix factorization w.r.t. $d$. Example 1 in the Section 4 of (Lin et al., 2001) shows that $\mathcal{S}_{1}$ is not empty, and Example 4.1 in the Section 4 of (Liu et al., 2011) shows that $\mathcal{S}_{1} \varsubsetneqq \mathcal{S}_{2}$.

Lemma 2.12 tell us that for any given $\mathbf{F} \in \mathcal{S}_{2}, \operatorname{rank}\left(\mathbf{F}\left(f\left(\mathbf{z}_{2}\right), \mathbf{z}_{2}\right)\right)=l-1$. This implies that $\operatorname{GCD}\left(d, d_{l-1}(\mathbf{F})\right)=1$. Otherwise, it follows from $d$ is an irreducible polynomial that $\operatorname{GCD}\left(d, d_{l-1}(\mathbf{F})\right)$ $=d$, then $c_{i}\left(f\left(\mathbf{z}_{2}\right), \mathbf{z}_{2}\right)=0(i=1, \ldots, \gamma)$ and $\operatorname{rank}\left(\mathbf{F}\left(f\left(\mathbf{z}_{2}\right), \mathbf{z}_{2}\right)\right)<l-1$, which leads to a contradiction. Now, we construct a new set of multivariate polynomial matrices:

$$
\mathcal{S}:=\left\{\mathbf{F} \in k[\mathbf{z}]^{l \times m}: d \mid d_{l}(\mathbf{F}) \text { and } \operatorname{GCD}\left(d, d_{l-1}(\mathbf{F})\right)=1\right\} .
$$

Then, $\emptyset \neq \mathcal{S}_{1} \varsubsetneqq \mathcal{S}_{2} \subset \mathcal{S}$. As we know, $d_{l-1}(\mathbf{F})$ is the GCD of $c_{1}, \ldots, c_{\gamma}$, then we have

$$
\left\langle d, c_{1}, \ldots, c_{\gamma}\right\rangle \subseteq\left\langle d, d_{l-1}(\mathbf{F})\right\rangle \subseteq k[\mathbf{z}] .
$$

Therefore, it follows that $\left\langle d, c_{1}, \ldots, c_{\gamma}\right\rangle \neq k[\mathbf{z}]$ if $\left\langle d, d_{l-1}(\mathbf{F})\right\rangle \neq k[\mathbf{z}]$. Although $\operatorname{GCD}\left(d, d_{l-1}(\mathbf{F})\right)=1$ for $\mathbf{F} \in \mathcal{S}, d$ and $d_{l-1}(\mathbf{F})$ may have common zeros. Next, we give an example to show that there exits $\mathbf{F} \in \mathcal{S} \backslash \mathcal{S}_{2}$ such that $\mathbf{F}$ admits a matrix factorization w.r.t. $d$.

Example 2.13. Let

$$
\mathbf{F}=\left[\begin{array}{ccc}
z_{1} z_{2}-z_{1}-z_{2}^{2}-z_{2} z_{3} & z_{1} z_{3}+z_{1}-z_{2} z_{3}-z_{2}-z_{3}^{2}-z_{3} & \mathbf{F}[1,3] \\
-z_{1} z_{2}-z_{1} z_{3}+z_{2}+z_{3} & z_{2}+z_{3} & z_{1} z_{2}+z_{1} z_{3} \\
z_{1} & -z_{1}+z_{2}+z_{3} & -2 z_{1}+z_{2}+z_{3}+1
\end{array}\right],
$$

where $\mathbf{F}[1,3]=-z_{1} z_{2}+z_{1} z_{3}+2 z_{1}+z_{2}^{2}-z_{2}-z_{3}^{2}-2 z_{3}-1$.

It is easy to compute that $d_{3}(\mathbf{F})=\left(z_{1}-z_{2}\right)\left(z_{2}+z_{3}\right)^{2}$ and $d_{2}(\mathbf{F})=z_{2}+z_{3}$. Let $d=z_{1}-z_{2}$, then $d \mid d_{3}(\mathbf{F})$ and $\operatorname{GCD}\left(d, d_{2}(\mathbf{F})\right)=1$. Hence, $\mathbf{F} \in \mathcal{S}$.

$a_{1}=\left(z_{1}-z_{2}\right)\left(z_{2}+z_{3}\right)^{2}$ is the $3 \times 3$ minor of $\mathbf{F}$, and extracting d from $a_{1}$ yields $e_{1}=\left(z_{2}+\right.$ $\left.z_{3}\right)^{2}$. It is easy to check that the reduced Gröbner basis of $\left\langle d, e_{1}\right\rangle$ w.r.t. the lexicographic order is $\left\{z_{1}-z_{2},\left(z_{2}+z_{3}\right)^{2}\right\}$, then $\mathbf{F} \notin \mathcal{S}_{1}$.

Since the reduced Gröbner basis of $\left\langle d, d_{2}(\mathbf{F})\right\rangle$ w.r.t. the lexicographic order is $\left\{z_{1}+z_{3}, z_{2}+z_{3}\right\}$, we have $\left\langle d, c_{1}, \ldots, c_{9}\right\rangle \subseteq\left\langle d, d_{2}(\mathbf{F})\right\rangle \neq k[\mathbf{z}]$. Then, $\mathbf{F} \notin \mathcal{S}_{2}$.

However, we can get a matrix factorization of $\mathbf{F}$ w.r.t. $d$ :

$$
\mathbf{F}=\left[\begin{array}{ccc}
d & 0 & -z_{3}-1 \\
0 & 1 & 0 \\
0 & 0 & 1
\end{array}\right]\left[\begin{array}{ccc}
z_{2}+z_{3} & 0 & -z_{2}-z_{3} \\
-z_{1} z_{2}-z_{1} z_{3}+z_{2}+z_{3} & z_{2}+z_{3} & z_{1} z_{2}+z_{1} z_{3} \\
z_{1} & -z_{1}+z_{2}+z_{3} & -2 z_{1}+z_{2}+z_{3}+1
\end{array}\right]
$$

In Example 2.13, we find that the reduced Gröbner basis of $\left\langle d, h_{1}, \ldots, h_{9}\right\rangle$ w.r.t. the lexicographic order is $\{1\}$. In spire of it, we consider the following question.

Question 2.14. Let $\mathbf{F} \in \mathcal{S}$. If $\left\langle d, h_{1}, \ldots, h_{\gamma}\right\rangle=k[\mathbf{z}]$, does $\mathbf{F}$ have a matrix factorization w.r.t. d? 


\section{Main Results}

Before giving the main theorem, we introduce two important lemmas.

Lemma 3.1 (Lin et al. (2001)). Let $g \in k[\mathbf{z}]$ and $f\left(\mathbf{z}_{2}\right) \in k\left[\mathbf{z}_{2}\right]$. If $g\left(f, z_{2}, \ldots, z_{n}\right)$ is a zero polynomial in $k\left[\mathbf{z}_{2}\right]$, then $\left(z_{1}-f\left(\mathbf{z}_{2}\right)\right)$ is a divisor of $g$.

The following lemma is a generalization of Lemma 2 in (Lin et al., 2001).

Lemma 3.2. Let $\mathbf{F} \in k[\mathbf{z}]^{l \times m}$ with $\operatorname{rank}(\mathbf{F})=l-1$. If $\left\langle h_{1}, \ldots, h_{\gamma}\right\rangle=k[\mathbf{z}]$, then there is a ZLP vector $\mathbf{w} \in k[\mathbf{z}]^{1 \times l}$ such that $\mathbf{w} \mathbf{F}=\mathbf{0}_{1 \times m}$.

Proof. In view of $\operatorname{rank}(\mathbf{F})=l-1$, we could assume that the first $(l-1)$ row vectors $\mathbf{f}_{1}, \ldots, \mathbf{f}_{l-1}$ of $\mathbf{F}$ are $k[\mathbf{z}]$-linearly independent. This implies that $\mathbf{f}_{1}, \ldots, \mathbf{f}_{l-1}$ and $\mathbf{f}_{l}$ are $k[\mathbf{z}]$-linearly dependent. Thus $\mathbf{w} \mathbf{F}=\mathbf{0}_{1 \times m}$ for some nonzero row vector $\mathbf{w}=\left[w_{1}, \ldots, w_{l}\right] \in k[\mathbf{z}]^{1 \times l}$, where $w_{l} \neq 0$ and $\operatorname{GCD}\left(w_{1}, \ldots, w_{l}\right)=1$. Obviously, $w_{1}, \ldots, w_{l}$ are all the $1 \times 1$ reduced minors of $\mathbf{w}$.

The next thing is to prove that $w_{1}, \ldots, w_{l}$ generate $k[\mathbf{z}]$. Let $\mathbf{F}_{1}, \ldots, \mathbf{F}_{\beta} \in k[\mathbf{z}]^{l \times(l-1)}$ be all the $l \times(l-1)$ submatrices of $\mathbf{F}$, where $\beta=\left(\begin{array}{c}m \\ l-1\end{array}\right)$. For each $1 \leq i \leq \beta$, let $c_{i 1}, \ldots, c_{i l}$ and $h_{i 1}, \ldots, h_{i l}$ be all the $(l-1) \times(l-1)$ minors and all the $(l-1) \times(l-1)$ reduced minors of $\mathbf{F}_{i}$ respectively, then $c_{i j}=d_{l-1}\left(\mathbf{F}_{i}\right) \cdot h_{i j}$, where $1 \leq j \leq l$. Let $\mathbf{w}=\left[\mathbf{w}_{1}, w_{l}\right]$, where $\mathbf{w}_{1}=\left[w_{1}, \ldots, w_{l-1}\right] \in k[\mathbf{z}]^{1 \times(l-1)}$. Let $\mathbf{F}_{i}=\left[\mathbf{F}_{i 1}^{\mathrm{T}},-\mathbf{F}_{i 2}^{\mathrm{T}}\right]^{\mathrm{T}}$, where $\mathbf{F}_{i 1} \in k[\mathbf{z}]^{(l-1) \times(l-1)}$ and $\mathbf{F}_{i 2} \in k[\mathbf{z}]^{1 \times(l-1)}$. If $\mathbf{F}_{i}$ is not of full column rank, then $c_{i j}=0$ and $h_{i j}=0, j=1, \ldots, l$. If $\mathbf{F}_{i}$ is of full column rank, then it follows from $\mathbf{w} \mathbf{F}=\mathbf{0}_{1 \times m}$ that

$$
\left[\mathbf{w}_{1}, w_{l}\right]\left[\begin{array}{c}
\mathbf{F}_{i 1} \\
-\mathbf{F}_{i 2}
\end{array}\right]=\mathbf{0}_{1 \times(l-1)} .
$$

Since $w_{l} \neq 0, \operatorname{det}\left(\mathbf{F}_{i 1}\right) \neq 0$ by Lemma 2.5. From equation (3.1) we have

$$
w_{l}^{-1} \mathbf{w}_{1}=\mathbf{F}_{i 2} \mathbf{F}_{i 1}^{-1} .
$$

According to Lemma 2.6, all the $1 \times 1$ reduced minors of $\mathbf{w}$ are equal to all the $(l-1) \times(l-1)$ reduced minors of $\mathbf{F}_{i}$ without considering the sign, i.e., $w_{j}=h_{i j}$ for $j=1, \ldots, l$. Therefore, all the $(l-1) \times(l-1)$ minors of $\mathbf{F}$ are as follows:

$$
d_{l-1}\left(\mathbf{F}_{1}\right) \cdot w_{1}, \ldots, d_{l-1}\left(\mathbf{F}_{1}\right) \cdot w_{l}, \cdots, d_{l-1}\left(\mathbf{F}_{\beta}\right) \cdot w_{1}, \ldots, d_{l-1}\left(\mathbf{F}_{\beta}\right) \cdot w_{l} .
$$

Let $\bar{d} \in k[\mathbf{z}]$ be the GCD of $d_{l-1}\left(\mathbf{F}_{1}\right), \ldots, d_{l-1}\left(\mathbf{F}_{\beta}\right)$, then there exists $\bar{d}_{i} \in k[\mathbf{z}]$ such that $d_{l-1}\left(\mathbf{F}_{i}\right)=$ $\bar{d} \cdot \bar{d}_{i}$, where $i=1, \ldots, \beta$. In the following we prove that the polynomials

$$
\begin{array}{cccc}
\bar{d}_{1} w_{1}, & \bar{d}_{1} w_{2}, & \cdots & \bar{d}_{1} w_{l}, \\
\bar{d}_{2} w_{1}, & \bar{d}_{2} w_{2}, & \cdots & \bar{d}_{2} w_{l}, \\
\vdots & \vdots & \ddots & \vdots \\
\bar{d}_{\beta} w_{1}, & \bar{d}_{\beta} w_{2}, & \cdots & \bar{d}_{\beta} w_{l},
\end{array}
$$

are all the $(l-1) \times(l-1)$ reduced minors of $\mathbf{F}$. It follows from $\operatorname{GCD}\left(w_{1}, \ldots, w_{l}\right)=1$ and $\operatorname{GCD}\left(\bar{d}_{1}, \cdots, \bar{d}_{\beta}\right)=1$ that

$$
\begin{aligned}
& \operatorname{GCD}\left(\bar{d}_{1} w_{1}, \ldots, \bar{d}_{1} w_{l}, \cdots, \bar{d}_{\beta} w_{1}, \ldots, \bar{d}_{\beta} w_{l}\right) \\
= & \operatorname{GCD}\left(\operatorname{GCD}\left(\bar{d}_{1} w_{1}, \ldots, \bar{d}_{1} w_{l}\right), \cdots, \operatorname{GCD}\left(\bar{d}_{\beta} w_{1}, \ldots, \bar{d}_{\beta} w_{l}\right)\right) \\
= & \operatorname{GCD}\left(\bar{d}_{1}, \cdots, \bar{d}_{\beta}\right) \\
= & 1 .
\end{aligned}
$$


Therefore, $\bar{d}_{1} w_{1}, \ldots, \bar{d}_{1} w_{l}, \cdots, \bar{d}_{\beta} w_{1}, \ldots, \bar{d}_{\beta} w_{l}$ are all the $(l-1) \times(l-1)$ reduced minors of $\mathbf{F}$, i.e., they are equal to $h_{1}, \ldots, h_{\gamma}$. Since $\left\langle h_{1}, \ldots, h_{\gamma}\right\rangle=k[\mathbf{z}], w_{1}, \ldots, w_{l}$ generate $k[\mathbf{z}]$.

Combining Lemma 3.1 and Lemma 3.2, we can answer Question 2.14.

Theorem 3.3. Let $\mathbf{F} \in \mathcal{S}$. If $\left\langle d, h_{1}, \ldots, h_{\gamma}\right\rangle=k[\mathbf{z}]$, then $\mathbf{F}$ admits a matrix factorization w.r.t. d.

Proof. We divide our proof into three steps.

First, let $\hat{\mathbf{F}}=\mathbf{F}\left(f\left(\mathbf{z}_{2}\right), \mathbf{z}_{2}\right) \in k\left[\mathbf{z}_{2}\right]^{l \times m}$, and we prove that $\operatorname{rank}(\hat{\mathbf{F}})=l-1$. Let $\hat{a}_{1}, \ldots, \hat{a}_{\eta} \in k\left[\mathbf{z}_{2}\right]$ and $\hat{c}_{1}, \ldots, \hat{c}_{\gamma} \in k\left[\mathbf{z}_{2}\right]$ be all the $l \times l$ minors and all the $(l-1) \times(l-1)$ minors of $\hat{\mathbf{F}}$, respectively. Then, $\hat{a}_{i}=a_{i}\left(f\left(\mathbf{z}_{2}\right), \mathbf{z}_{2}\right)$ and $\hat{c}_{j}=c_{j}\left(f\left(\mathbf{z}_{2}\right), \mathbf{z}_{2}\right)$, where $1 \leq i \leq \eta$ and $1 \leq j \leq \gamma$. Since $\mathbf{F} \in \mathcal{S}$, we have $d \mid d_{l}(\mathbf{F})$ and $\operatorname{GCD}\left(d, d_{l-1}(\mathbf{F})\right)=1 . d \mid d_{l}(\mathbf{F})$ implies that $\hat{a}_{i}=a_{i}\left(f\left(\mathbf{z}_{2}\right), \mathbf{z}_{2}\right)=0 \quad(i=1, \ldots, \eta)$ and $\operatorname{rank}(\hat{\mathbf{F}}) \leq l-1$. If $\operatorname{rank}(\hat{\mathbf{F}})<l-1$, then $c_{j}\left(f\left(\mathbf{z}_{2}\right), \mathbf{z}_{2}\right)=\hat{c}_{j}=0 \quad(j=1, \ldots, \gamma)$. It follows from Lemma 3.1 that $d$ is a common divisor of $c_{1}, \ldots, c_{\gamma}$, then $d \mid d_{l-1}(\mathbf{F})$, which contradicts $\operatorname{GCD}\left(d, d_{l-1}(\mathbf{F})\right)=1$. Therefore, $\operatorname{rank}(\hat{\mathbf{F}})=l-1$.

Second, we prove that all the $(l-1) \times(l-1)$ reduced minors of $\hat{\mathbf{F}}$ generate $k\left[\mathbf{z}_{2}\right]$. Let $\bar{h} \in k\left[\mathbf{z}_{2}\right]$ be the GCD of $h_{1}\left(f\left(\mathbf{z}_{2}\right), \mathbf{z}_{2}\right), \ldots, h_{\gamma}\left(f\left(\mathbf{z}_{2}\right), \mathbf{z}_{2}\right)$, then for each $1 \leq j \leq \gamma$ there exits $\hat{h}_{j} \in k\left[\mathbf{z}_{2}\right]$ such that $h_{j}\left(f\left(\mathbf{z}_{2}\right), \mathbf{z}_{2}\right)=\bar{h} \cdot \hat{h}_{j}$, and $\operatorname{GCD}\left(\hat{h}_{1}, \ldots, \hat{h}_{\gamma}\right)=1$. Let $g=d_{l-1}(\mathbf{F})$, then it follows from $\hat{c}_{j}=g\left(f\left(\mathbf{z}_{2}\right), \mathbf{z}_{2}\right) \cdot h_{j}\left(f\left(\mathbf{z}_{2}\right), \mathbf{z}_{2}\right)$ that $d_{l-1}(\hat{\mathbf{F}})=g\left(f\left(\mathbf{z}_{2}\right), \mathbf{z}_{2}\right) \cdot \bar{h}$, and $\hat{h}_{1}, \ldots, \hat{h}_{\gamma}$ are all the $(l-1) \times(l-1)$ reduced minors of $\hat{\mathbf{F}}$. Assume that $\left\langle\hat{h}_{1}, \ldots, \hat{h}_{\gamma}\right\rangle \neq k\left[\mathbf{z}_{2}\right]$, then there exists a point $\left(\alpha_{2}, \ldots, \alpha_{n}\right) \in k^{1 \times(n-1)}$ such that $\hat{h}_{j}\left(\alpha_{2}, \ldots, \alpha_{n}\right)=0$, where $j=1, \ldots, \gamma$. Let $\alpha_{1}=f\left(\alpha_{2}, \ldots, \alpha_{n}\right)$, then for each $j$ we have $h_{j}\left(\alpha_{1}, \alpha_{2}, \ldots, \alpha_{n}\right)=\bar{h}\left(\alpha_{2}, \ldots, \alpha_{n}\right) \cdot \hat{h}_{j}\left(\alpha_{2}, \ldots, \alpha_{n}\right)=0$. This implies that $\left(\alpha_{1}, \alpha_{2}, \ldots, \alpha_{n}\right) \in k^{1 \times n}$ is a common zero of $d, h_{1}, \ldots, h_{\gamma}$, which contradicts the fact that $\left\langle d, h_{1}, \ldots, h_{\gamma}\right\rangle=k[\mathbf{z}]$.

Finally, we remark that $\mathbf{F}$ has a matrix factorization w.r.t. $d$. Using Lemma 3.2, we get $\mathbf{w} \hat{\mathbf{F}}=\mathbf{0}_{1 \times m}$, where $\mathbf{w} \in k\left[\mathbf{z}_{2}\right]^{1 \times l}$ is a ZLP vector. Meanwhile, according to Lemma 2.10, a unimodular matrix $\mathbf{U} \in k\left[\mathbf{z}_{2}\right]^{l \times l}$ can be constructed such that $\mathbf{w}$ is its first row. Let $\mathbf{F}_{0}=\mathbf{U F}$, then the first row of $\mathbf{F}_{0}\left(f\left(\mathbf{z}_{2}\right), \mathbf{z}_{2}\right)=\mathbf{U} \hat{\mathbf{F}}$ is a zero vector. By Lemma 3.1, $d$ is a common divisor of the polynomials in the first row of $\mathbf{F}_{0}$, thus

$$
\mathbf{F}_{0}=\mathbf{U F}=\mathbf{D F}_{1}=\left[\begin{array}{ccccc}
d & & & \\
& 1 & & \\
& & \ddots & \\
& & & 1
\end{array}\right]\left[\begin{array}{ccc}
\bar{f}_{11} & \cdots & \bar{f}_{1 m} \\
f_{21} & \cdots & f_{2 m} \\
\vdots & \ddots & \vdots \\
f_{l 1} & \cdots & f_{l m}
\end{array}\right] \text {. }
$$

Consequently, we can now derive the matrix factorization of $\mathbf{F}$ w.r.t. $d$ :

$$
\mathbf{F}=\mathbf{G}_{1} \mathbf{F}_{1}
$$

where $\mathbf{G}_{1}=\mathbf{U}^{-1} \mathbf{D} \in k[\mathbf{z}]^{l \times l}, \mathbf{F}_{1} \in k[\mathbf{z}]^{l \times m}$ and $\operatorname{det}\left(\mathbf{G}_{1}\right)=d$.

Remark 3.4. In Theorem 3.3, we have that $\operatorname{rank}(\hat{\mathbf{F}})=l-1$ and $\left\langle\hat{h}_{1}, \ldots, \hat{h}_{\gamma}\right\rangle=k\left[\mathbf{z}_{2}\right]$. Hence, Theorem 3.3 is a generalization of Lemma 2.12.

According to Theorem 3.3, we construct a set of multivariate polynomial matrices:

$$
\mathcal{S}_{3}:=\left\{\mathbf{F} \in \mathcal{S}:\left\langle d, h_{1}, \ldots, h_{\gamma}\right\rangle=k[\mathbf{z}]\right\} .
$$


Then, $\mathcal{S}_{2} \subset \mathcal{S}_{3} \subset \mathcal{S}$ and $\mathbf{F} \in \mathcal{S}_{3}$ admits a matrix factorization w.r.t. $d$. Example 2.13 in Section 2.2 shows that $S_{2} \varsubsetneqq S_{3}$.

Let $\mathbf{F} \in k[\mathbf{z}]^{l \times m}$, and $d_{0}=\prod_{t=1}^{s}\left(z_{1}-f_{t}\left(\mathbf{z}_{2}\right)\right)$ be a divisor of $d_{l}(\mathbf{F})$, where $f_{1}\left(\mathbf{z}_{2}\right), \ldots, f_{s}\left(\mathbf{z}_{2}\right) \in$ $k\left[\mathbf{z}_{2}\right]$. Liu et al. (2011) proved that if $\left\langle d_{0}, c_{1}, \ldots, c_{\gamma}\right\rangle=k[\mathbf{z}]$, then $\mathbf{F}$ admits a matrix factorization w.r.t. $d_{0}$. It would be interesting to know whether Theorem 3.3 can be generalized to the case with $t>1$. Without loss of generality, we consider the case of $t=2$.

Theorem 3.5. Let $\mathbf{F} \in k[\mathbf{z}]^{l \times m}$ and $d_{0}=\left(z_{1}-f_{1}\left(\mathbf{z}_{2}\right)\right)\left(z_{1}-f_{2}\left(\mathbf{z}_{2}\right)\right)$ be a divisor of $d_{l}(\mathbf{F})$. If $\operatorname{GCD}\left(d_{0}, d_{l-1}(\mathbf{F})\right)=1$ and $\left\langle d_{0}, h_{1}, \ldots, h_{\gamma}\right\rangle=k[\mathbf{z}]$, then $\mathbf{F}$ admits a matrix factorization w.r.t. $d_{0}$.

Proof. Let $d_{1}=z_{1}-f_{1}\left(\mathbf{z}_{2}\right)$ and $d_{2}=z_{1}-f_{2}\left(\mathbf{z}_{2}\right)$. Obviously, $\operatorname{GCD}\left(d_{1}, d_{l-1}(\mathbf{F})\right)=1$ and $\left\langle d_{1}, h_{1}, \ldots, h_{\gamma}\right\rangle=k[\mathbf{z}]$. By Theorem 3.3, there exist $\mathbf{G}_{1} \in k[\mathbf{z}]^{l \times l}$ and $\mathbf{F}_{1} \in k[\mathbf{z}]^{l \times m}$ such that

$$
\mathbf{F}=\mathbf{G}_{1} \mathbf{F}_{1},
$$

where $\mathbf{G}_{1}=\mathbf{U}_{1}^{-1} \mathbf{D}_{1}, \operatorname{det}\left(\mathbf{G}_{1}\right)=d_{1}, \mathbf{U}_{1} \in k\left[\mathbf{z}_{2}\right]^{l \times l}$ is a unimodular matrix and $\mathbf{D}_{1}=\operatorname{diag}\left(d_{1}, 1, \ldots, 1\right)$. According the Equation (2.2) in Lemma $2.3, d_{2}=z_{1}-f_{2}\left(\mathbf{z}_{2}\right)$ is a divisor of $d_{l}\left(\mathbf{F}_{1}\right)$. Next we prove that $\mathbf{F}_{1}$ admits a matrix factorization w.r.t. $d_{2}$.

We first prove that $\operatorname{GCD}\left(d_{2}, d_{l-1}\left(\mathbf{F}_{1}\right)\right)=1$. Otherwise, it follows from $d_{2}$ is an irreducible polynomial that $\operatorname{GCD}\left(d_{2}, d_{l-1}\left(\mathbf{F}_{1}\right)\right)=d_{2}$. Then $d_{l-1}\left(\mathbf{F}_{1}\right) \mid d_{l-1}(\mathbf{F})$ implies that $d_{2} \mid d_{l-1}(\mathbf{F})$, which contradicts the condition $\operatorname{GCD}\left(d, d_{l-1}(\mathbf{F})\right)=1$. Second, we prove that $d_{2}$ and all the $(l-1) \times(l-1)$ reduced minors of $\mathbf{F}_{1}$ generate the unit ideal $k[\mathbf{z}]$.

Let $\mathbf{F}_{i 1} \in k[\mathbf{z}]^{(l-1) \times m}$ be a submatrix obtained by removing the $i$-th row of $\mathbf{F}_{1}$, and $\bar{c}_{i 1}, \ldots, \bar{c}_{i \beta}$ be all the $(l-1) \times(l-1)$ minors of $\mathbf{F}_{i 1}$, where $i=1, \ldots, l$. Then, $\bar{c}_{11}, \ldots, \bar{c}_{1 \beta}, \ldots, \bar{c}_{l 1}, \ldots, \bar{c}_{l \beta}$ are all the $(l-1) \times(l-1)$ minors of $\mathbf{F}_{1}$. Extracting $d_{l-1}\left(\mathbf{F}_{1}\right)$ from $\bar{c}_{i j}$ yields $\bar{c}_{i j}=d_{l-1}\left(\mathbf{F}_{1}\right) \cdot \bar{h}_{i j}$, then $\bar{h}_{11}, \ldots, \bar{h}_{1 \beta}, \ldots, \bar{h}_{l 1}, \ldots, \bar{h}_{l \beta}$ are all the $(l-1) \times(l-1)$ reduced minors of $\mathbf{F}_{1}$. Hence, we only need to prove that $\left\langle d_{2}, \bar{h}_{11}, \ldots, \bar{h}_{l \beta}\right\rangle=k[\mathbf{z}]$.

Since $\mathbf{D}_{1}=\operatorname{diag}\left(d_{1}, 1, \ldots, 1\right)$, all the $(l-1) \times(l-1)$ minors of $\mathbf{D}_{1} \mathbf{F}_{1}$ are

$$
\bar{c}_{11}, \ldots, \bar{c}_{1 \beta}, d_{1} \bar{c}_{21}, \ldots, d_{1} \bar{c}_{2 \beta}, \ldots, d_{1} \bar{c}_{l 1}, \ldots, d_{1} \bar{c}_{l \beta}
$$

Obviously, there is at least one integer $j \in\{1, \ldots, \beta\}$ such that $d_{1} \nmid \bar{c}_{1 j}$. Otherwise, $d_{1} \mid d_{l-1}\left(\mathbf{D}_{1} \mathbf{F}_{1}\right)$. It follows form $\mathbf{F}=\mathbf{U}_{1}^{-1} \mathbf{D}_{1} \mathbf{F}_{1}$ and the Equation (2.1) in Lemma 2.3 that $d_{l-1}\left(\mathbf{D}_{1} \mathbf{F}_{1}\right) \mid d_{l-1}(\mathbf{F})$. So $d_{1} \mid d_{l-1}(\mathbf{F})$, which leads to a contradiction. Since $d_{1}=z_{1}-f_{1}\left(\mathbf{z}_{2}\right)$ is an irreducible polynomial, we have

$$
\begin{aligned}
& \operatorname{GCD}\left(\bar{c}_{11}, \ldots, \bar{c}_{1 \beta}, d_{1} \bar{c}_{21}, \ldots, d_{1} \bar{c}_{2 \beta}, \ldots, d_{1} \bar{c}_{l 1}, \ldots, d_{1} \bar{c}_{l \beta}\right) \\
= & \operatorname{GCD}\left(\bar{c}_{11}, \ldots, \bar{c}_{1 \beta}, \bar{c}_{21}, \ldots, \bar{c}_{2 \beta}, \ldots, \bar{c}_{l 1}, \ldots, \bar{c}_{l \beta}\right),
\end{aligned}
$$

Therefore, $d_{l-1}\left(\mathbf{D}_{1} \mathbf{F}_{1}\right)=d_{l-1}\left(\mathbf{F}_{1}\right)$. It follows from $\mathbf{U}_{1} \mathbf{F}=\mathbf{D}_{1} \mathbf{F}_{1}$ that $d_{l-1}(\mathbf{F}) \mid d_{l-1}\left(\mathbf{D}_{1} \mathbf{F}_{1}\right)$ and $d_{l-1}(\mathbf{F})=d_{l-1}\left(\mathbf{F}_{1}\right)$. The Equation (2.1) in Lemma 2.3 implies that each $c_{i}$ is a $k[\mathbf{z}]$-linear combination of $\bar{c}_{11}, \ldots, \bar{c}_{l \beta}$, where $i=1, \ldots, \gamma$. Since $d_{l-1}(\mathbf{F})=d_{l-1}\left(\mathbf{F}_{1}\right)$, we can obtain that each $h_{i}(1 \leq i \leq \gamma)$ is a $k[\mathbf{z}]$-linear combination of $\bar{h}_{11}, \ldots, \bar{h}_{l \beta}$. By $\left\langle d_{0}, h_{1}, \ldots, h_{\gamma}\right\rangle=k[\mathbf{z}]$, $\left\langle d_{2}, h_{1}, \ldots, h_{\gamma}\right\rangle=k[\mathbf{z}]$. If $\left\langle d_{2}, \bar{h}_{11}, \ldots, \bar{h}_{l \beta}\right\rangle \neq k[\mathbf{z}]$, then there exits a point $\left(\alpha_{1}, \ldots, \alpha_{n}\right) \in k^{1 \times n}$ such that $\bar{h}_{i j}\left(\alpha_{1}, \ldots, \alpha_{n}\right)=0$ for each $i$ and $j$, where $\alpha_{1}=f_{2}\left(\alpha_{2}, \ldots, \alpha_{n}\right)$. This implies that $\left(\alpha_{1}, \ldots, \alpha_{n}\right)$ is a common zero of $d_{2}, h_{1}, \ldots, h_{\gamma}$, which leads to a contradiction. 
According to Theorem 3.3 again, there exits $\mathbf{G}_{2} \in k[\mathbf{z}]^{l \times l}$ and $\mathbf{F}_{2} \in k[\mathbf{z}]^{l \times m}$ such that $\mathbf{F}_{1}=$ $\mathbf{G}_{2} \mathbf{F}_{2}$, where $\mathbf{G}_{2}=\mathbf{U}_{2}^{-1} \mathbf{D}_{2}$, $\operatorname{det}\left(\mathbf{G}_{2}\right)=d_{2}, \mathbf{U}_{2} \in k\left[\mathbf{z}_{2}\right]^{l \times l}$ is an unimodular matrix and $\mathbf{D}_{2}=$ $\operatorname{diag}\left(d_{2}, 1, \ldots, 1\right)$.

Finally, we can get a matrix factorization of $\mathbf{F}$ w.r.t. $d_{0}$ :

$$
\mathbf{F}=\mathbf{G}_{0} \mathbf{F}_{2},
$$

where $\mathbf{G}_{0}=\mathbf{G}_{1} \mathbf{G}_{2} \in k[\mathbf{z}]^{l \times l}$, and $\operatorname{det}\left(\mathbf{G}_{0}\right)=d_{0}=\left(z_{1}-f_{1}\left(\mathbf{z}_{2}\right)\right)\left(z_{1}-f_{2}\left(\mathbf{z}_{2}\right)\right)$.

Remark 3.6. In the above theorem, we can factorize $\mathbf{F}_{1}$ w.r.t. $d_{2}$ without checking whether $\operatorname{GCD}\left(d_{2}, d_{l-1}\left(\mathbf{F}_{1}\right)\right)=1$ and the ideal generated by $d_{2}$ and all the $(l-1) \times(l-1)$ reduced minors of $\mathbf{F}_{1}$ is $k[\mathbf{z}]$, which can help us improve the computational efficiency of matrix factorizations.

It is worth noting that if $f_{1}\left(\mathbf{z}_{2}\right)=f_{2}\left(\mathbf{z}_{2}\right)$ in Theorem 3.5, we have the following corollary.

Corollary 3.7. Let $\mathbf{F} \in k[\mathbf{z}]^{l \times m}$ and $d_{0}=\left(z_{1}-f_{1}\left(\mathbf{z}_{2}\right)\right)^{r}$ be a divisor of $d_{l}(\mathbf{F})$. If $\operatorname{GCD}\left(d_{0}, d_{l-1}(\mathbf{F})\right)=$ 1 and $\left\langle d_{0}, h_{1}, \ldots, h_{\gamma}\right\rangle=k[\mathbf{z}]$, then $\mathbf{F}$ admits a matrix factorization w.r.t. $d_{0}$.

Further, if $f_{1}\left(\mathbf{z}_{2}\right) \neq f_{2}\left(\mathbf{z}_{2}\right)$ in Theorem 3.5, we have another corollary.

Corollary 3.8. Let $\mathbf{F} \in k[\mathbf{z}]^{l \times m}$ and $d_{0}=\prod_{t=1}^{s}\left(z_{1}-f_{t}\left(\mathbf{z}_{2}\right)\right)^{q_{t}}$ be a divisor of $d_{l}(\mathbf{F})$. If $\operatorname{GCD}\left(d_{0}, d_{l-1}(\mathbf{F})\right)$ $=1$ and $\left\langle d_{0}, h_{1}, \ldots, h_{\gamma}\right\rangle=k[\mathbf{z}]$, then $\mathbf{F}$ admits a matrix factorization w.r.t. $d_{0}$.

Let $f^{(i)}(\mathbf{z})$ be a polynomial in $k\left[z_{1}, \ldots, z_{i-1}, z_{i+1}, \ldots, z_{n}\right]$, where $1 \leq i \leq n$. Similarly, we can get the following corollaries.

Corollary 3.9. Let $\mathbf{F} \in k[\mathbf{z}]^{l \times m}$ and $d_{0}=\left(z_{i}-f^{(i)}(\mathbf{z})\right)^{r}$ be a divisor of $d_{l}(\mathbf{F})$. If $\operatorname{GCD}\left(d_{0}, d_{l-1}(\mathbf{F})\right)=$ 1 and $\left\langle d_{0}, h_{1}, \ldots, h_{\gamma}\right\rangle=k[\mathbf{z}]$, then $\mathbf{F}$ admits a matrix factorization w.r.t. $d_{0}$.

Corollary 3.10. Let $\mathbf{F} \in k[\mathbf{z}]^{l \times m}$ and $d_{0}=\prod_{i=1}^{n} \prod_{t=1}^{s_{i}}\left(z_{i}-f_{t}^{(i)}(\mathbf{z})\right)^{q_{i t}}$ be a divisor of $d_{l}(\mathbf{F})$. If $\operatorname{GCD}\left(d_{0}, d_{l-1}(\mathbf{F})\right)=1$ and $\left\langle d_{0}, h_{1}, \ldots, h_{\gamma}\right\rangle=k[\mathbf{z}]$, then $\mathbf{F}$ admits a matrix factorization w.r.t. $d_{0}$.

\section{Algorithm and Example}

According to Theorem 3.3, we get the following algorithm for computing a matrix factorization of $\mathbf{F} \in \mathcal{S}_{3}$ w.r.t. $d$.

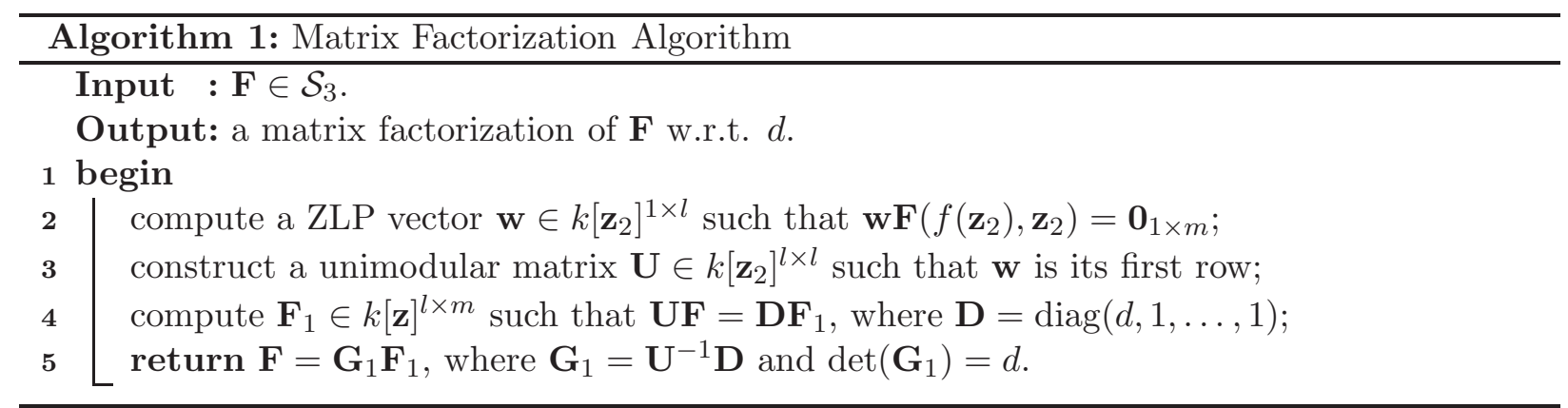

In the following, we show how to compute $\mathbf{w}$ and $\mathbf{U}$ in Algorithm 1. Let $\hat{\mathbf{F}}=\mathbf{F}\left(f\left(\mathbf{z}_{2}\right), \mathbf{z}_{2}\right) \in$ $k\left[\mathbf{z}_{2}\right]^{l \times m}$ and $\operatorname{Syz}_{l}(\hat{\mathbf{F}})$ be the left syzygy module of $\hat{\mathbf{F}}$, i.e., $\operatorname{Syz}_{l}(\hat{\mathbf{F}})=\left\{\mathbf{p} \in k\left[\mathbf{z}_{2}\right]^{1 \times l} \mid \mathbf{p} \hat{\mathbf{F}}=\mathbf{0}_{1 \times m}\right\}$. 
Since $\operatorname{rank}(\hat{\mathbf{F}})=l-1$, we have $\operatorname{rank}\left(\operatorname{Syz}_{l}(\hat{\mathbf{F}})\right)=1$. Then, we compute a reduced Gröbner basis of $\operatorname{Syz}_{l}(\hat{\mathbf{F}})$ w.r.t. a term order, and select a nonzero vector from the Gröbner basis. Let $\mathbf{w}_{1}=$ $\left[w_{11}, \ldots, w_{1 l}\right] \in k\left[\mathbf{z}_{2}\right]^{1 \times l}$ be the nonzero vector, and $w \in k\left[\mathbf{z}_{2}\right]$ be the GCD of $w_{11}, \ldots, w_{1 l}$, then $\mathbf{w}=\frac{\mathbf{w}_{1}}{w}$.

Since $\mathbf{w}$ is a ZLP vector, there exists a column vector $\mathbf{q}_{1} \in k\left[\mathbf{z}_{2}\right]^{l \times 1}$ such that $\mathbf{w q}_{1}=1$. This calculation problem is equivalent to a lifting homomorphism problem in (Decker and Lossen, 2006) (see Problem 4.1, page 129), and the command "lift" of the computer algebra system Singular in (Decker et al., 2016) can help us compute $\mathbf{q}_{1}$. Let $\operatorname{Syz}_{r}(\mathbf{w})=\left\{\mathbf{q} \in k\left[\mathbf{z}_{2}\right]^{l \times 1} \mid \mathbf{w q}=0\right\}$, then $\operatorname{Syz}_{r}(\mathbf{w})$ is a free module with $\operatorname{rank}\left(\operatorname{Syz}_{r}(\mathbf{w})\right)=(l-1)$ by Lemma 2.9. Let $\mathbf{q}_{2}, \ldots, \mathbf{q}_{l} \in k\left[\mathbf{z}_{2}\right]^{l \times 1}$ be a free basis of $\operatorname{Syz}_{r}(\mathbf{w})$, then $\mathbf{V}=\left[\mathbf{q}_{1}, \mathbf{q}_{2}, \ldots, \mathbf{q}_{l}\right] \in k\left[\mathbf{z}_{2}\right]^{l \times l}$ is a unimodular matrix and $\mathbf{U}=\mathbf{V}^{-1}$ is one that we want by Theorem 4.4 in (Lu et al., 2017).

Now, we use an example to illustrate the calculation process of Algorithm 1. We return to Example 2.13, and let $\mathbf{F}$ be the same matrix in Example 2.13.

Example 4.1. Let

$$
\mathbf{F}=\left[\begin{array}{ccc}
z_{1} z_{2}-z_{1}-z_{2}^{2}-z_{2} z_{3} & z_{1} z_{3}+z_{1}-z_{2} z_{3}-z_{2}-z_{3}^{2}-z_{3} & \mathbf{F}[1,3] \\
-z_{1} z_{2}-z_{1} z_{3}+z_{2}+z_{3} & z_{2}+z_{3} & z_{1} z_{2}+z_{1} z_{3} \\
z_{1} & -z_{1}+z_{2}+z_{3} & -2 z_{1}+z_{2}+z_{3}+1
\end{array}\right],
$$

where $\mathbf{F}[1,3]=-z_{1} z_{2}+z_{1} z_{3}+2 z_{1}+z_{2}^{2}-z_{2}-z_{3}^{2}-2 z_{3}-1$.

As already noted in Example 2.13, $\left\langle d, h_{1}, \ldots, h_{9}\right\rangle=k\left[z_{1}, z_{2}, z_{3}\right]$ implies that $\mathbf{F} \in \mathcal{S}_{3}$. Then, we can use Algorithm 1 to factorize $\mathbf{F}$ w.r.t. d.

Step 1: Let $\hat{\mathbf{F}}=\mathbf{F}\left(z_{2}, z_{2}, z_{3}\right) \in k\left[z_{2}, z_{3}\right]^{3 \times 3}$, we compute a ZLP vector $\mathbf{w} \in k\left[z_{2}, z_{3}\right]^{1 \times 3}$ such that $\mathbf{w} \hat{\mathbf{F}}=\mathbf{0}_{1 \times 3}$, where

$$
\hat{\mathbf{F}}=\left[\begin{array}{ccc}
-z_{2}\left(z_{3}+1\right) & -z_{3}\left(z_{3}+1\right) & -\left(z_{3}-z_{2}+1\right)\left(z_{3}+1\right) \\
\left(1-z_{2}\right)\left(z_{2}+z_{3}\right) & z_{2}+z_{3} & z_{2}\left(z_{2}+z_{3}\right) \\
z_{2} & z_{3} & z_{3}-z_{2}+1
\end{array}\right] .
$$

We use Singular command "syz" to compute a reduced Gröbner basis of $\operatorname{Syz}_{l}(\hat{\mathbf{F}})$ w.r.t. the lexicographic order, and obtain $\mathbf{w}=\left[1,0, z_{3}+1\right]$.

Step 2: Construct a unimodular matrix $\mathbf{U} \in k\left[z_{2}, z_{3}\right]^{3 \times 3}$ such that $\mathbf{w}$ is its first row. According to the instruction of the construction for unimodular matrix $\mathbf{U}$ below Algorithm 1, we divide it into three small steps.

Step 2.1: Using Singular command "lift" to compute $\mathbf{q}_{1} \in k\left[z_{2}, z_{3}\right]^{3 \times 1}$ such that $\mathbf{w q}_{1}=1$, we get $\mathbf{q}_{1}=[1,0,0]^{\mathrm{T}}$.

Step 2.2: Using QuillenSuslin package to compute a free basis of $\operatorname{Syz}_{r}(\mathbf{w})$, we have $\mathbf{q}_{2}=[0,1,0]^{\mathrm{T}}$ and $\mathbf{q}_{3}=\left[-\left(z_{3}+1\right), 0,1\right]^{\mathrm{T}}$.

Step 2.3: Let $\mathbf{V}=\left[\mathbf{q}_{1}, \mathbf{q}_{2}, \mathbf{q}_{3}\right]$, then

$$
\mathbf{U}=\mathbf{V}^{-1}=\left[\begin{array}{ccc}
1 & 0 & z_{3}+1 \\
0 & 1 & 0 \\
0 & 0 & 1
\end{array}\right]
$$

Step 3. Extracting $d$ from the first row of $\mathbf{U F}$, we get $\mathbf{U F}=\mathbf{D F}_{1}$, where $\mathbf{D}=\operatorname{diag}(d, 1,1)$ and

$$
\mathbf{F}_{1}=\left[\begin{array}{ccc}
z_{2}+z_{3} & 0 & -z_{2}-z_{3} \\
-z_{1} z_{2}-z_{1} z_{3}+z_{2}+z_{3} & z_{2}+z_{3} & z_{1} z_{2}+z_{1} z_{3} \\
z_{1} & -z_{1}+z_{2}+z_{3} & -2 z_{1}+z_{2}+z_{3}+1
\end{array}\right] .
$$


Then, we obtain a matrix factorization of $\mathbf{F}$ w.r.t. d:

$$
\mathbf{F}=\mathbf{G F}_{1}=\left[\begin{array}{ccc}
d & 0 & -z_{3}-1 \\
0 & 1 & 0 \\
0 & 0 & 1
\end{array}\right]\left[\begin{array}{ccc}
z_{2}+z_{3} & 0 & -z_{2}-z_{3} \\
-z_{1} z_{2}-z_{1} z_{3}+z_{2}+z_{3} & z_{2}+z_{3} & z_{1} z_{2}+z_{1} z_{3} \\
z_{1} & -z_{1}+z_{2}+z_{3} & -2 z_{1}+z_{2}+z_{3}+1
\end{array}\right],
$$

where $\mathbf{G}=\mathbf{U}^{-1} \mathbf{D}$ and $\operatorname{det}\left(\mathbf{G}_{1}\right)=d=z_{1}-z_{2}$.

\section{Conclusions}

We have studied the problem of matrix factorizations for multivariate polynomial matrices in $\mathcal{S}$, and the results presented in this paper greatly extend those of (Lin et al., 2001; Liu et al., 2011). The matrix factorizations for an arbitrary multivariate polynomial matrix remains a challenging and an important open problem. Although the new results can only deal with the class of multivariate polynomial matrices discussed in $\mathcal{S}$, we hope that the new results will motivate new progress in this important research topic.

Acknowledgements This research was supported by the CAS Project QYZDJ-SSW-SYS022.

\section{References}

Bose, N., 1982. Applied multidimensional systems theory. Van Nostrand Reinhold, New York.

Bose, N., Buchberger, B., Guiver, J., 2003. Multidimensional systems theory and applications. Dordrecht, The Netherlands: Kluwer.

Buchberger, B., 1965. Ein Algorithmus zum Auffinden der Basiselemente des Restklassenrings nach einem nulldimensionalen Polynomideal. Ph.D. thesis, Universitat Innsbruck, Austria.

Charoenlarpnopparut, C., Bose, N., 1999. Multidimensional FIR filter bank design using Gröbner bases. IEEE Transactions on Circuits and Systems II: Analog. Digit. Signal Processing 46 (12), 1475-1486.

Cox, D., Little, J., O'shea, D., 2007. Ideals, varieties, and algorithms. Undergraduate Texts in Mathematics. Springer, New York, third edition.

Decker, W., Greuel, G.-M., Pfister, G., Schoenemann, H., 2016. SINGULAR 4.0.3. a computer algebra system for polynomial computations, FB Mathematik der Universitaet, D-67653 Kaiserslautern. https://www. singular.uni-kl.de/.

Decker, W., Lossen, C., 2006. Computing in Algebraic Geometry. Algorithms and Computation in Mathematics. Springer Berlin Heidelberg.

Fabiańska, A., Quadrat, A., 2006. Applications of the Qullen-Suslin theorem to multidimensional systems theory. In Gröbner Bases in Control Theory and Signal Processing, edited by Park, H. and Regensburger, G., Walter de Gruyter, Berlin., 23-106.

Fabiańska, A., Quadrat, A., 2007. A Maple implementation of a constructive version of the Quillen-Suslin Theorem. https://wwwb.math.rwth-aachen.de/QuillenSuslin/.

Guan, J., Li, W., Ouyang, B., 2018. On rank factorizations and factor prime factorizations for multivariate polynomial matrices. Journal of Systems Science and Complexity 31 (6), 1647-1658.

Guan, J., Li, W., Ouyang, B., 2019. On minor prime factorizations for multivariate polynomial matrices. Multidimensional Systems and Signal Processing 30, 493-502.

Guiver, J., Bose, N., 1982. Polynomial matrix primitive factorization over arbitrary coefficient field and related results. IEEE Transactions on Circuits and Systems 29 (10), 649-657.

Lin, Z., 1988. On matrix fraction descriptions of multivariable linear n-D systems. IEEE Transactions on Circuits and Systems 35 (10), 1317-1322.

Lin, Z., 1993. On primitive factorizations for n-D polynomial matrices. In: IEEE International Symposium on Circuits and Systems. pp. 601-618.

Lin, Z., 1999a. Notes on n-D polynomial matrix factorizations. Multidimensional Systems and Signal Processing 10 (4), 379-393.

Lin, Z., 1999b. On syzygy modules for polynomial matrices. Linear Algebra and Its Applications 298 (1-3), 73-86. 
Lin, Z., 2001. Further results on n-D polynomial matrix factorizations. Multidimensional Systems and Signal Processing 12 (2), 199-208.

Lin, Z., Bose, N., 2001. A generalization of Serre's conjecture and some related issues. Linear Algebra and Its Applications 338 (1), 125-138.

Lin, Z., Ying, J., Xu, L., 2001. Factorizations for n-D polynomial matrices. Circuits, Systems, and Signal Processing 20 (6), 601-618.

Liu, J., Li, D., Wang, M., 2011. On general factorizations for n-D polynomial matrices. Circuits Systems and Signal Processing 30 (3), 553-566.

Liu, J., Li, D., Zheng, L., 2014. The Lin-Bose problem. IEEE Transactions on Circuits and Systems II Express Briefs $61(1), 41-43$.

Liu, J., Wang, M., 2010. Notes on factor prime factorizations for n-D polynomial matrices. Multidimensional Systems and Signal Processing 21 (1), 87-97.

Liu, J., Wang, M., 2013. New results on multivariate polynomial matrix factorizations. Linear Algebra and Its Applications 438 (1), 87-95.

Liu, J., Wang, M., 2015. Further remarks on multivariate polynomial matrix factorizations. Linear Algebra and Its Applications 465 (465), 204-213.

Logar, A., Sturmfels, B., 1992. Algorithms for the Quillen-Suslin theorem. Journal of Algebra 145 (1), $231-239$.

Lu, D., Ma, X., Wang, D., 2017. A new algorithm for general factorizations of multivariate polynomial matrices. In: proceedings of International Symposium on Symbolic and Algebraic Computation. pp. 277-284.

Morf, M., Levy, B., Kung, S., 1977. New results in 2-D systems theory, part i: 2-D polynomial matrices, factorization, and coprimeness. Proceedings of the IEEE 64 (6), 861-872.

Park, H., 1995. A computational theory of Laurent polynomial rings and multidimensional FIR systems. Ph.D. thesis, University of California at Berkeley.

Pommaret, J., 2001. Solving Bose conjecture on linear multidimensional systems. In: European Control Conference. IEEE, Porto, Portugal, pp. 1653-1655.

Quillen, D., 1976. Projective modules over polynomial rings. Inventiones mathematicae 36 (1), 167-171.

Serre, J., 1955. Faisceaux algébriques cohérents. Annals of Mathematics 61 (2), 197-278.

Strang, G., 2010. Linear algebra and its applications. Academic Press.

Suslin, A., 1976. Projective modules over polynomial rings are free. Soviet Math. Dokl. 17, 1160-1164.

Wang, M., 2007. On factor prime factorization for n-D polynomial matrices. IEEE Transactions on Circuits and Systems 54 (6), 1398-1405.

Wang, M., Feng, D., 2004. On Lin-Bose problem. Linear Algebra and Its Applications 390 (1), 279-285.

Wang, M., Kwong, C., 2005. On multivariate polynomial matrix factorization problems. Mathematics of Control, Signals, and Systems 17 (4), 297-311.

Youla, D., Gnavi, G., 1979. Notes on n-dimensional system theory. IEEE Transactions on Circuits and Systems $26(2), 105-111$

Youla, D., Pickel, P., 1984. The Quillen-Suslin theorem and the structure of n-dimensional elementary polynomial matrices. IEEE Transactions on Circuits and Systems 31 (6), 513-518. 